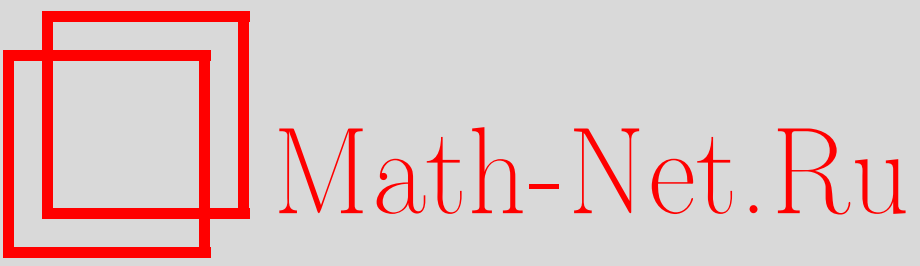

В. С. Дрюма, К теории пространств постоянной кривизны, ТМФ, 2006, том 146, номер 1, 42-54

DOI: https://doi.org/10.4213/tmf2007

Использование Общероссийского математического портала Math-Net.Ru подразумевает, что вы прочитали и согласны с пользовательским соглашением

http://www . mathnet.ru/rus/agreement

Параметры загрузки:

IP : 34.227 .88 .159

26 апреля 2023 г., 12:43:20 
ТЕОРЕТИЧЕСКАЯ

И МАТЕМАТИЧЕСКАЯ

ФИЗИКА

Том 146, № 1

январь, 2006

(C) 2006 г.

В. С. Дрюма*

\section{К ТЕОРИИ ПРОСТРАНСТВ ПОСТОЯННОЙ КРИВИЗНЫ}

Построен ряд примеров трехмерных метрик постоянной кривизны, определяемых решениями нелинейных интегрируемых дифференциальных уравнений и их обобщений. Исследованы свойства римановых расширений метрик постоянной кривизны. Рассмотрена связь с теорией нормальных римановых пространств.

Ключевые слова: римановы расширения, пространства постоянной кривизны, уравнение Кортевега-де Фриза.

\section{1. ВВЕДЕНИЕ}

Метрики постоянной кривизны определяются следующими условиями на тензор кривизны:

$$
R_{i j k l}-\lambda\left(g_{i k} g_{j l}-g_{i l} g_{j k}\right)=0 .
$$

В случае диагональной метрики (здесь и далее $(\vec{r})=(x, y, x))$

$$
d s^{2}=A^{2}(\vec{r}) d x^{2}+B^{2}(\vec{r}) d y^{2}+C^{2}(\vec{r}) d z^{2}
$$

условия (1) эквивалентны системе дифференциальных уравнений на функции $A, B$, $C$, которые имеют вид

$$
\begin{aligned}
& \partial_{y z}^{2} A(\vec{r})=\frac{\partial_{z} B(\vec{r}) \partial_{y} A(\vec{r})}{B(\vec{r})}+\frac{\partial_{y} C(\vec{r}) \partial_{z} A(\vec{r})}{C(\vec{r})} \\
& \partial_{x z}^{2} B(\vec{r})=\frac{\partial_{z} A(\vec{r}) \partial_{x} B(\vec{r})}{A(\vec{r})}+\frac{\partial_{x} C(\vec{r}) \partial_{z} B(\vec{r})}{C(\vec{r})} \\
& \partial_{x y}^{2} C(\vec{r})=\frac{\partial_{y} A(\vec{r}) \partial_{x} C(\vec{r})}{A(\vec{r})}+\frac{\partial_{x} B(\vec{r}) \partial_{y} C(\vec{r})}{B(\vec{r})}
\end{aligned}
$$

*Institute of Mathematics and Informatics AS Moldova, Kishinev, Moldova. E-mail: valery@dryuma.com, cainar@mail.md 


$$
\begin{aligned}
& \lambda C(\vec{r}) B(\vec{r})+\frac{\partial_{x} C(\vec{r}) \partial_{x} B(\vec{r})}{A^{2}(\vec{r})}+\frac{\partial_{z}^{2} B(\vec{r})}{C(\vec{r})}- \\
&- \frac{\partial_{z} B(\vec{r}) \partial_{z} C(\vec{r})}{C^{2}(\vec{r})}+\frac{\partial_{y}^{2} C(\vec{r})}{B(\vec{r})}-\frac{\partial_{y} B(\vec{r}) \partial_{y} C(\vec{r})}{B^{2}(\vec{r})}=0 \\
& \lambda A(\vec{r}) C(\vec{r})-\frac{\partial_{z} A(\vec{r}) \partial_{z} C(\vec{r})}{C^{2}(\vec{r})}+\frac{\partial_{z}^{2} A(\vec{r})}{C(\vec{r})}+ \\
& \quad+\frac{\partial_{y} A(\vec{r}) \partial_{y} C(\vec{r})}{B^{2}(\vec{r})}+\frac{\partial_{x}^{2} C(\vec{r})}{A(\vec{r})}-\frac{\partial_{x} A(\vec{r}) \partial_{x} C(\vec{r})}{A^{2}(\vec{r})}=0 \\
& \lambda B(\vec{r}) A(\vec{r})-\frac{\partial_{x} A(\vec{r}) \partial_{x} B(\vec{r})}{A^{2}(\vec{r})}+\frac{\partial_{x}^{2} B(\vec{r})}{A(\vec{r})}+ \\
& \quad+\frac{\partial_{z} A(\vec{r}) \partial_{z} B(\vec{r})}{C^{2}(\vec{r})}+\frac{\partial_{y}^{2} A(\vec{r})}{B(\vec{r})}-\frac{\partial_{y} A(\vec{r}) \partial_{y} B(\vec{r})}{B^{2}(\vec{r})}=0 .
\end{aligned}
$$

Здесь и далее мы используем обозначения

$$
\partial_{x} \equiv \frac{\partial}{\partial x}, \quad \partial_{x x}^{2} \equiv \frac{\partial^{2}}{\partial x \partial x}, \quad \partial_{x y}^{2} \equiv \frac{\partial^{2}}{\partial x \partial y}
$$

и т.д. Решения полной системы уравнений (2)-(7) зависят от значений кривизны пространства $\lambda$ и играют важную роль в геометрии трехмерных пространств.

Заметим, что первые три уравнения не зависят от параметра $\lambda$, и их решения являются общими для всех возможных уравнений, описывающих метрики пространств постоянной кривизны.

Система уравнений (2)-(4) как пример точно интегрируемой системы многомерных уравнений была найдена в работе [1] и исследовалась в работах [2] наряду с применением этих уравнений в задаче о триортогональной системе поверхностей в пространствах $\mathbb{R} P^{3}$ и $\mathbb{R}^{3}$.

\section{2. ПРОСТРАНСТВО НУЛЕВОЙ КРИВИЗНЫ $\lambda=0$}

Свойства пространств нулевой кривизны $\lambda=0$ в диагональной метрике были исследованы в работе [3]. Здесь мы рассмотрим метрику недиагонального вида [4]

$$
\begin{array}{rl}
d s^{2}=y^{2} & d x^{2}+2\left(l(x, z) y^{2}+m(x, z)\right) d x d z+2 d y d z+ \\
& +\left(l^{2}(x, z) y^{2}-2\left(\partial_{x} l(x, z)\right) y+2 l(x, z) m(x, z)+2 l(x, z)\right) d z^{2}
\end{array}
$$

с некоторыми функциями $l(x, z)$ и $m(x, z)$. 
Условие на тензор кривизны $R_{i j k l}=0$ для метрики (8) приводит к соотношениям

$$
\begin{aligned}
R_{1313}=( & \left.\partial_{x}^{3} l(x, z)-3 l(x, z) \partial_{x} l(x, z)+\partial_{z} l(x, z)\right) y+ \\
& +\left(-l(x, z) \partial_{x}^{2} m(x, z)+\partial_{x z}^{2} m(x, z)-\right. \\
& \left.-3 \partial_{x} l(x, z) \partial_{x} m(x, z)-2 m(x, z) \partial_{x}^{2} l(x, z)-\partial_{x}^{2} l(x, z)\right)- \\
& -\frac{1}{y}\left(-m(x, z) \partial_{z} m(x, z)+m(x, z) l(x, z) \partial_{x} m(x, z)+\right. \\
& \left.+\partial_{x} l(x, z) m(x, z)+2 \partial_{x} l(x, z) m^{2}(x, z)\right)=0, \\
R_{1323}=\frac{1}{y} & \left(\partial_{x} l(x, z)-\partial_{z} m(x, z)+2 \partial_{x} l(x, z) m(x, z)+l(x, z) \partial_{x} m(x, z)\right)=0,
\end{aligned}
$$

которые эквивалентны системе уравнений для функций $l(x, z)$ и $m(x, z)$

$$
\begin{aligned}
& \partial_{x}^{3} l(x, z)-3 l(x, z) \partial_{x} l(x, z)+\partial_{z} l(x, z)=0, \\
& \partial_{x} l(x, z)-\partial_{z} m(x, z)+2 \partial_{x} l(x, z) m(x, z)+l(x, z) \partial_{x} m(x, z)=0 .
\end{aligned}
$$

Таким образом, может быть сформулирована следующая

ТеОрема 1. Существует класс трехмерных плоских метрик, определяемый решениями системы уравнений (9), (10).

Отметим, что первое из уравнений системы - известное уравнение Кортевегаде Фриза $(К д \Phi)$, и это обстоятельство может быть использовано для изучения свойств ортогональных метрик.

Рассмотрим некоторые примеры:

1) $l(x, z)=-x /(3 z), \quad m(x, z)=-1 / 2+F_{1}\left(z / x^{3}\right) x^{-2}$

2) $l(x, z)=-4(\operatorname{ch}(x-4 z))^{-2}, m(x, z)=-1 / 2$.

ЗАмЕЧАНИЕ 1. В простейшем случае трехмерные метрики нулевой кривизны имеют вид

$$
\begin{array}{rl}
d s^{2}=y^{2} & d x^{2}+2\left(l(x, z) y^{2}-\frac{1}{2}\right) d x d z+2 d y d z+ \\
& +\left(l^{2}(x, z) y^{2}-2 \partial_{x} l(x, z) y+l(x, z)\right) d z^{2}
\end{array}
$$

где функция $l(x, z)$ является решением классического уравнения КдФ

$$
\partial_{z} l(x, z)-3 l(x, z) \partial_{x} l(x, z)+\partial_{x}^{3} l(x, z)=0 .
$$

В частности, функции

$$
l(x, z)=-4(\operatorname{ch}(x-4 z))^{-2}
$$

и

$$
l(x, z)=-24 \frac{4 \operatorname{ch}(2 x-8 z)+\operatorname{ch}(4 x-64 z)+3}{(3 \operatorname{ch}(x-28 z)+\operatorname{ch}(3 x-36 z))^{2}}
$$


доставляют примеры метрик подобного типа.

Несмотря на то что определитель метрик не зависит от функции $l(x, z)$, их свойства можно охарактеризовать с помощью уравнения на собственные значения для оператора Лапласа, определенного на 1-формах,

$$
A(\vec{r})=A_{i}(\vec{r}) d x^{i}
$$

которое имеет вид

$$
g^{i j} \nabla_{i} \nabla_{j} A_{k}-R_{k}^{l} A_{l}=-\lambda A_{k} .
$$

В частном случае $l(x, z)=0$ и $A_{i}(\vec{r})=[h(y), q(y), f(y)]$ эти уравнения принимают вид

$$
\begin{gathered}
-\frac{1}{4 y^{3}}\left[h^{\prime}(y)-4 f^{\prime}(y) y^{2}-h^{\prime \prime}(y) y+4 \lambda h(y) y^{3}\right]=0 \\
-\frac{1}{4 y^{4}}\left[-6 h(y)-3 q(y)+3 q^{\prime}(y) y+4 h^{\prime}(y) y-\right. \\
\left.\quad-q^{\prime \prime}(y) y^{2}+4 f(y) y^{2}+4 \lambda q(y) y^{4}\right]=0 \\
-\frac{1}{4 y^{3}}\left[f^{\prime}(y)-f^{\prime \prime}(y) y+4 \lambda f(y) y^{3}\right]=0 .
\end{gathered}
$$

Решения этой системы зависят от собственных значений $\lambda$ и характеризуют свойства соответствующих плоских метрик.

ЗАмЕчАниЕ 2. В теории многообразий характеристический класс Черна-Саймонса строится из матричной калибровочной связности $A_{j k}^{i}$ следующим образом:

$$
W(A)=\frac{1}{4 \pi^{2}} \int d^{3} x \epsilon^{i j k} \operatorname{tr}\left(\frac{1}{2} A_{i} \partial_{j} A_{k}+\frac{1}{3} A_{i} A_{j} A_{k}\right)
$$

Этот член можно превратить в трехмерную геометрическую величину, заменив матричную связность $A_{j k}^{i}$ на связность Кристоффеля $\Gamma_{j k}^{i}$.

Для плотности инварианта Черна-Саймонса можно получить выражение [5]

$$
C S(\Gamma)=\epsilon^{i j k}\left(\Gamma_{i q}^{p} \Gamma_{k p ; j}^{q}+\frac{2}{3} \Gamma_{i q}^{p} \Gamma_{j r}^{q} \Gamma_{k p}^{r}\right)
$$

Для метрики (8) находим

$$
\begin{aligned}
C S(\Gamma)=- & \frac{5 l(x, z) \partial_{x} l(x, z)-5 \partial_{z} l(x, z)}{\sqrt{y^{2}}}- \\
& \quad-\frac{3 l(x, z) \partial_{x} m(x, z)-4\left(\partial_{x} l(x, z)\right) m(x, z)-3 \partial_{z} m(x, z)-2 \partial_{x} l(x, z)}{y^{2} \sqrt{y^{2}}} .
\end{aligned}
$$

Используя эту формулу, получаем для первого примера

$$
C S(\Gamma)=\frac{10}{9} \frac{x \operatorname{sign}(y)}{z^{2} y}-\frac{10}{3} F_{1}\left(\frac{z}{x^{3}}\right) \operatorname{sign}(y) z^{-1} x^{-2} y^{-3}
$$


и для второго примера

$$
C S(\Gamma)=160 \frac{\operatorname{ch}(x-4 z)(\operatorname{sh}(x-4 z))^{3} \operatorname{sign}(y)}{y} .
$$

\section{3. МЕТРИКИ НЕНУЛЕВОЙ КРИВИЗНЫ $\lambda \neq 0$}

Метрика пространства положительной кривизны $\lambda=1$ определяется формулой

$$
d s^{2}=\frac{d x^{2}+d y^{2}+d z^{2}}{\left(1+\left(x^{2}+y^{2}+z^{2}\right) / 4\right)^{2}},
$$

а метрика пространства отрицательной кривизны $\lambda=-1$ имеет вид

$$
d s^{2}=\frac{d x^{2}+d y^{2}+d z^{2}}{z^{2}} .
$$

Исходя из этих выражений можно получить более общие примеры метрик постоянной кривизны.

Например, подстановка выражений

$$
A(\vec{r})=\frac{1}{z}, \quad B(\vec{r})=\frac{1}{z}+v(z), \quad C(\vec{r})=\frac{1}{z}+u(z)
$$

в систему $(2)-(7)$ и интегрирование соответствующих уравнений для функций $u(z)$ и $v(z)$ дает метрику

$$
d s^{2}=\frac{d x^{2}}{z^{2}}+\left(z^{-2}-1\right) d y^{2}+\frac{d z^{2}}{z^{2}\left(1-z^{2}\right)}
$$

отрицательной кривизны $\lambda=-1$.

Подстановка

$$
A(\vec{r})=\left(1+\frac{1}{4}\left(x^{2}+y^{2}+z^{2}\right)\right)^{-1}+U(\vec{r})
$$

в систему (2)-(7) и интегрирование соответствующих уравнений приводит к метрикам

$$
d s^{2}=A^{2}(\vec{r}) d x^{2}+\frac{d y^{2}+d z^{2}}{\left(1+\left(x^{2}+y^{2}+z^{2}\right) / 4\right)^{2}}
$$

с функцией $A(\vec{r})$ вида

$$
A(\vec{r})=4 \frac{\sqrt{x^{2}\left(x^{2}-4 \lambda+4\right)}}{\left(x^{2}-4 \lambda+4\right)\left(4+x^{2}+y^{2}+z^{2}\right)} .
$$

Инвариант Черна-Саймонса ортогональной трехмерной метрики с условиями (2)-(4) на компоненты дается выражением

$$
C S(\Gamma)=\frac{10}{A(\vec{r}) B(\vec{r}) C(\vec{r})}\left[\partial_{y} A(\vec{r}) \partial_{z} B(\vec{r}) \partial_{x} C(\vec{r})-\partial_{z} A(\vec{r}) \partial_{x} B(\vec{r}) \partial_{y} C(\vec{r})\right]
$$


После подстановки компонент метрики (13) в это выражение получаем

$$
C S(\Gamma)=0
$$

Как следует из (14), класс трехмерных ортогональных метрик с обращающимся в нуль инвариантом Черна-Саймонса определяется условием

$$
\partial_{z} A(\vec{r}) \partial_{x} B(\vec{r}) \partial_{y} C(\vec{r})-\partial_{y} A(\vec{r}) \partial_{z} B(\vec{r}) \partial_{x} C(\vec{r})=0
$$

Метрики вида

$$
d s^{2}=2 E(\vec{r}) d x d y+d z^{2}
$$

являются метриками постоянной кривизны, если функция $E(\vec{r})$ определяется выражением

$$
E(\vec{r})=\frac{1}{4} \frac{(F(x, y) \sin (\sqrt{\lambda} z)-F(x, y) \cos (\sqrt{\lambda} z))^{2}}{\lambda}
$$

где

$$
-4 \partial_{y} F(x, y) \partial_{x} F(x, y)+4 F(x, y) \partial_{x y}^{2} F(x, y)+F^{4}(x, y)=0
$$

или после подстановки $F(x, y)=e^{U(x, y)}$

$$
4 \partial_{x y}^{2} U(x, y)+e^{2 U(x, y)}=0 .
$$

Последнее уравнение - это известное уравнение Лиувилля с общим решением

$$
U(x, y)=\frac{1}{2} \ln \left(-4 \frac{a^{\prime}(x) b^{\prime}(y)}{\left(a^{2}(x)+b^{2}(y)\right)^{2}}\right) .
$$

Инвариант Черна-Саймонса для таких метрик обращается в нуль.

Рассмотрим метрики вида

$$
d s^{2}=d x^{2}+2 \cos u(x, y) d x d y+A^{2}(x, y) d z^{2} .
$$

Условия (1) приводят к линейной системе уравнений

$$
\begin{aligned}
& \partial_{x y}^{2} A(x, y)+\lambda A(x, y) \cos u(x, y)=0 \\
& \partial_{x}^{2} A(x, y)-\frac{\cos u(x, y) \partial_{x} u(x, y) \partial_{x} A(x, y)}{\sin u(x, y)}+ \\
&+\lambda A(x, y)+\frac{\partial_{x} u(x, y) \partial_{y} A(x, y)}{\sin u(x, y)}=0 \\
& \partial_{y}^{2} A(x, y)-\frac{\cos u(x, y) \partial_{y} u(x, y) \partial_{y} A(x, y)}{\sin u(x, y)}+ \\
&+\lambda A(x, y)+\frac{\partial_{x} A(x, y) \partial_{y} u(x, y)}{\sin u(x, y)}=0
\end{aligned}
$$


которая совместна на решениях уравнения синус-Гордон $(\lambda=-\mu)$

$$
\partial_{x y}^{2} u(x, y)+\lambda \sin u(x, y)=0
$$

Таким образом, для любого решения уравнения синус-Гордон можно найти функцию $A(x, y)$, решив соответствующую линейную систему уравнений.

Рассмотрим пример. Простейшее решение уравнения (16) при $\lambda=-1$ дается формулой

$$
u(x, y)=4 \operatorname{arctg}\left(e^{x+y}\right)
$$

В этом случае линейная система принимает вид

$$
\begin{aligned}
& \partial_{x y}^{2} A(x, y)+\frac{\left(6 e^{2 x+2 y}-1-e^{4 x+4 y}\right) A(x, y)}{\left(1+e^{2 x+2 y}\right)^{2}}=0 \\
& \left(1-e^{4 x+4 y}\right) A(x, y)+\left(-2 e^{2 x+2 y}-e^{4 x+4 y}-1\right) \partial_{y} A(x, y)+ \\
& \quad+\left(-6 e^{2 x+2 y}+1+e^{4 x+4 y}\right) \partial_{x} A(x, y)+\left(-1+e^{4 x+4 y}\right) \partial_{x}^{2} A(x, y)=0 \\
& \left(1-e^{4 x+4 y}\right) A(x, y)+\left(-6 e^{2 x+2 y}+1+e^{4 x+4 y}\right) \partial_{y} A(x, y)+ \\
& \quad+\left(-2 e^{2 x+2 y}-e^{4 x+4 y}-1\right) \partial_{x} A(x, y)+\left(-1+e^{4 x+4 y}\right) \partial_{y}^{2} A(x, y)=0
\end{aligned}
$$

а ее решение есть

$$
A(x, y)=\frac{e^{x+y}}{1+e^{2 x+2 y}}
$$

\section{4. ПРИМЕРЫ СУЩЕСТВЕННО ТРЕХМЕРНЫХ МЕТРИК}

Рассмотрим семейство трехмерных метрик вида

$$
d s^{2}=d x^{2}+2 \cos B(\vec{r}) d x d y+d y^{2}+\left(\partial_{z} B(\vec{r})\right)^{2} d z^{2}
$$

Условия (1) для таких метрик приводят к следующей системе уравнений:

$$
\begin{aligned}
\partial_{x y}^{2} B(\vec{r}) & +\frac{1}{4} \sin B(\vec{r})(-1+4 \lambda)=0 \\
\partial_{y y z}^{3} B(\vec{r}) & -\frac{\cos B(\vec{r}) \partial_{y} B(\vec{r}) \partial_{y z}^{2} B(\vec{r})}{\sin B(\vec{r})}+ \\
& +\frac{\partial_{x z}^{2} B(\vec{r}) \partial_{y} B(\vec{r})}{\sin B(\vec{r})}-\left(\frac{1}{4}-\lambda\right) \partial_{z} B(\vec{r})=0, \\
\partial_{x x z}^{3} B(\vec{r}) & -\frac{\cos B(\vec{r}) \partial_{x} B(\vec{r}) \partial_{x z}^{2} B(\vec{r})}{\sin B(\vec{r})}+ \\
& +\frac{\partial_{x} B(\vec{r}) \partial_{y z}^{2} B(\vec{r})}{\sin B(\vec{r})}-\left(\frac{1}{4}-\lambda\right) \partial_{z} B(\vec{r})=0 .
\end{aligned}
$$

Эта система совместна, и ее решения дают примеры трехмерных метрик постоянной кривизны. 
Член Черна-Саймонса в этом случае определяется выражением

$$
C S(\Gamma)=-\frac{5}{2} \partial_{x z}^{2} B(\vec{r}) \partial_{y} B(\vec{r})+\frac{5}{2} \partial_{x} B(\vec{r}) \partial_{y z}^{2} B(\vec{r})
$$

Рассмотрим пример. Для простоты представим метрику в эквивалентном виде

$$
d s^{2}=d x^{2}+2 u(\vec{r}) d x d y+d y^{2}+\frac{\left(\partial_{z} u(\vec{r})\right)^{2} d z^{2}}{1-u^{2}(\vec{r})},
$$

где функция $u(\vec{r})$ определяется из условия

$$
u(\vec{r})=\arccos (B(\vec{r})) .
$$

Система уравнений для функции $B(\vec{r})$ переходит при этом в более простую систему для функции $u(\vec{r})$, интегрирование которой при условии $\lambda=1 / 4$ приводит к метрике

$$
d s^{2}=d x^{2}+\frac{1}{2} \frac{\left(z^{4} f^{2}(x) h^{2}(y)+1\right) d x d y}{z^{2} f(x) h(y)}+d y^{2}-4 \frac{d z^{2}}{z^{2}},
$$

где $f(x), h(y)$ - произвольные функции. Тензор Риччи в этом случае равен

$$
\mathrm{R}_{a b}=\left[\begin{array}{ccc}
\frac{1}{2} & \frac{1}{4} \frac{z^{4} f^{2}(x) h^{2}(y)+1}{z^{2} f(x) h(y)} & 0 \\
\frac{1}{4} \frac{z^{4} f^{2}(x) h^{2}(y)+1}{z^{2} f(x) h(y)} & \frac{1}{2} & 0 \\
0 & 0 & -2 z^{-2}
\end{array}\right]
$$

Инвариант Черна-Саймонса этой метрики равен нулю.

ЗАмЕЧАниЕ 3. Абсолютное значение кривизны $\lambda=1 / 4$ является особым значением в теории римановых многообразий и связано с теорией многообразий защемленной кривизны.

\section{5. РИМАНОВЫ РАСШИРЕНИЯ ПРОСТРАНСТВ ПОСТОЯННОЙ КРИВИЗНЫ $\lambda= \pm 1,0$}

Пространство с диагональной метрикой имеет следующие коэффициенты связности:

$$
\begin{array}{lll}
\Gamma_{11}^{1}=\frac{\partial_{x} A(\vec{r})}{A(\vec{r})}, & \Gamma_{11}^{2}=-\frac{A(\vec{r}) \partial_{y} A(\vec{r})}{B^{2}(\vec{r})} \\
\Gamma_{11}^{3}=-\frac{A(\vec{r}) \partial_{z} A(\vec{r})}{C^{2}(\vec{r})}, & \Gamma_{12}^{1}=\frac{\partial_{y} A(\vec{r})}{A(\vec{r})}, \quad \Gamma_{12}^{2}=\frac{\partial_{x} B(\vec{r})}{B(\vec{r})} \\
\Gamma_{12}^{3}=0, \quad \Gamma_{13}^{1}=\frac{\partial_{z} A(\vec{r})}{A(\vec{r})}, & \Gamma_{13}^{2}=0, \quad \Gamma_{13}^{3}=\frac{\partial_{x} C(\vec{r})}{C(\vec{r})} \\
\Gamma_{22}^{1}=-\frac{B(\vec{r}) \partial_{x} B(\vec{r})}{A^{2}(\vec{r})}, & \Gamma_{22}^{2}=\frac{\partial_{y} B(\vec{r})}{B(\vec{r})}
\end{array}
$$


50

$$
\begin{array}{ll}
\Gamma_{22}^{3}=-\frac{B(\vec{r}) \partial_{z} B(\vec{r})}{C^{2}(\vec{r})}, & \Gamma_{23}^{1}=0, \quad \Gamma_{23}^{2}=\frac{\partial_{z} B(\vec{r})}{B(\vec{r})}, \\
\Gamma_{23}^{3}=\frac{\partial_{y} C(\vec{r})}{C(\vec{r})}, & \Gamma_{33}^{1}=-\frac{C(\vec{r}) \partial_{x} C(\vec{r})}{A^{2}(\vec{r})}, \\
\Gamma_{33}^{2}=-\frac{C(\vec{r}) \partial_{y} C(\vec{r})}{B^{2}(\vec{r})}, & \Gamma_{33}^{3}=\frac{\partial_{z} C(\vec{r})}{C(\vec{r})} .
\end{array}
$$

В. С. ДРЮМА

Используя эти выражения, введем шестимерную метрику

$$
{ }^{6} d s^{2}=-2 \Gamma_{j k}^{i} d x^{j} d x^{k} \psi_{i}+2 d x^{i} d \psi_{i},
$$

где $\psi_{1}, \psi_{2}, \psi_{3}$ - некоторые дополнительные координаты [6].

Тензор Риччи метрики (18) обращается в нуль при выполнении условий (2)-(7), $\lambda=0$, и тензор Римана ${ }^{6} R_{i j k l}$ также обращается в нуль в этом случае. Таким образом, в результате риманова расширения плоской трехмерной диагональной метрики $\lambda=0$ с помощью коэффициентов (17) мы получаем шестимерное плоское пространство ${ }^{6} R_{i j k l}=0$ с сигнатурой [+++---].

Тензор Риччи метрики (1) при условиях (2)-(7) имеет компоненты

$$
\begin{gathered}
R_{11}=2 \lambda A^{2}(\vec{r}), \quad R_{22}=2 \lambda B^{2}(\vec{r}), \quad R_{33}=2 \lambda C^{2}(\vec{r}), \\
R_{12}=0, \quad R_{13}=0, \quad R_{23}=0,
\end{gathered}
$$

и соответствующее пространство в случае $\lambda= \pm 1$ является симметричным со следующими условиями на тензор кривизны:

$$
{ }^{3} R_{i j k l ; m}=0
$$

Замечательное обстоятельство состоит в том, что в результате риманова расширения пространства постоянной кривизны мы получаем симметричное пространство.

Теорема 2. Риманово расширение трехмерного пространства постоянной кривизны представляет собой шестимерное симметричное пространство

$$
{ }^{6} R_{i j k l ; m}=0
$$

Данное утверждение может быть доказано прямым вычислением.

ЗАмечАниЕ 4. В случае $\Gamma_{23}^{1} \neq 0, \Gamma_{12}^{3} \neq 0, \Gamma_{13}^{2} \neq 0$ можно получить шестимерную искривленную метрику

$$
{ }^{6} d s^{2}=-2 \Gamma_{j k}^{i} d x^{j} d x^{k} \psi_{i}+2 d x^{i} d \psi_{i}
$$

с условиями

$$
\Gamma_{13}^{2}=\frac{C(\vec{r}) A(\vec{r})}{B(\vec{r})}, \quad \Gamma_{12}^{3}=\frac{A(\vec{r}) B(\vec{r})}{C(\vec{r})}, \quad \Gamma_{23}^{1}=\frac{B(\vec{r}) C(\vec{r})}{A(\vec{r})}
$$


для пространств кривизны $\lambda=1$ и условиями

$$
\Gamma_{13}^{2}=\frac{i C(\vec{r}) A(\vec{r})}{B(\vec{r})}, \quad \Gamma_{12}^{3}=\frac{i A(\vec{r}) B(\vec{r})}{C(\vec{r})}, \quad \Gamma_{23}^{1}=\frac{i B(\vec{r}) C(\vec{r})}{A(\vec{r})}
$$

для пространств кривизны $\lambda=-1$. Для коэффициентов связности такого вида мы получаем обобщение системы уравнений Дарбу

$$
\begin{aligned}
\partial_{y z}^{2} A(\vec{r})= & \frac{\partial_{z} B(\vec{r}) \partial_{y} A(\vec{r})}{B(\vec{r})}+\frac{\partial_{y} C(\vec{r}) \partial_{z} A(\vec{r})}{C(\vec{r})}+ \\
& +2 \partial_{x}(B(\vec{r}) C(\vec{r})), \\
\partial_{x z}^{2} B(\vec{r})= & \frac{\partial_{z} A(\vec{r}) \partial_{x} B(\vec{r})}{A(\vec{r})}+\frac{\partial_{x} C(\vec{r}) \partial_{z} B(\vec{r})}{C(\vec{r})}+ \\
& +2 \partial_{y}(A(\vec{r}) C(\vec{r})), \\
\partial_{x y}^{2} C(\vec{r})= & \frac{\partial_{y} A(\vec{r}) \partial_{x} C(\vec{r})}{A(\vec{r})}+\frac{\partial_{x} B(\vec{r}) \partial_{y} C(\vec{r})}{B(\vec{r})}+ \\
& +2 \partial_{z}(A(\vec{r}) B(\vec{r})),
\end{aligned}
$$

которое может быть полезно в приложениях.

Например, простейшим решением этой системы является решение

$$
A(\vec{r})=e^{z / 4-x}, \quad B(\vec{r})=e^{y-z / 4}, \quad C(\vec{r})=e^{z / 4},
$$

которое соответствует метрике (19) шестимерного многообразия с соответствующими условиями на тензор кривизны.

\section{6. НОРМАЛЬНЫЕ РИМАНОВЫ ПРОСТРАНСТВА}

Понятие нормального риманова пространства было впервые введено Эйзенхартом. Свойства таких пространств были исследованы в работе [7].

ОПРЕДЕЛЕНИЕ. $n$-Мерное риманово пространство $N\left(x^{k}\right)$ с метрикой

$$
d s^{2}=g_{i j} d x^{i} d x^{j}
$$

является нормальным, если выполнены следующие условия на главные кривизны $K_{i}$ :

$$
\partial_{x^{l}} K_{l}=3 \lambda_{l}+3 \mu_{l} K_{l}, \quad i=l,
$$

и

$$
\begin{aligned}
\partial_{x^{l}} K_{i} & =\lambda_{l}+\mu_{l} K_{i}, & & i \neq l, \\
\partial_{x^{l}} \ln g_{i j} & =\frac{2}{K_{l}-K_{i}} \partial_{x^{l}} K_{i}, & & i \neq l,
\end{aligned}
$$

где $\lambda$ и $\mu$ - функции координат $x^{k}$. 
ЗАмЕчАниЕ 5 . Значения $K_{i}$ суть корни алгебраического уравнения

$$
\left|b_{i j}-\kappa g_{i j}\right|=0
$$

относительно тензора второго ранга $b_{i j}$, определенного в данном римановом пространстве.

В трехмерном случае главные кривизны $K_{i}$ удовлетворяют системе уравнений

$$
\begin{aligned}
& \left(K_{2}-K_{3}\right) \partial_{x} K_{1}+3\left(K_{3}-K_{1}\right) \partial_{x} K_{2}+3\left(K_{1}-K_{2}\right) \partial_{x} K_{3}=0 \\
& 3\left(K_{2}-K_{3}\right) \partial_{y} K_{1}+\left(K_{3}-K_{1}\right) \partial_{y} K_{2}+3\left(K_{1}-K_{2}\right) \partial_{y} K_{3}=0 \\
& 3\left(K_{2}-K_{3}\right) \partial_{z} K_{1}+3\left(K_{3}-K_{1}\right) \partial_{z} K_{2}+\left(K_{1}-K_{2}\right) \partial_{z} K_{3}=0 .
\end{aligned}
$$

Связи между системами уравнений Дарбу (2)-(4) и системой (21) были впервые установлены в работе [8].

Теорема 3. Система уравнений (21) для главных кривизн трехмерных нормальных пространств превращается в систему уравнений

$$
\begin{aligned}
\partial_{x y}^{2} K_{1}(\vec{r}) & +\frac{\partial_{y} A(\vec{r}) \partial_{x} K_{1}(\vec{r})}{A(\vec{r})}+ \\
& +\left(\frac{\partial_{x} A(\vec{r})}{A(\vec{r})}+\frac{\partial_{x} B(\vec{r})}{B(\vec{r})}-\frac{\partial_{x y}^{2} A(\vec{r})}{\partial_{y} A(\vec{r})}\right) \partial_{y} K_{1}(\vec{r})=0 \\
\partial_{x z}^{2} K_{1}(\vec{r}) & +\frac{\partial_{z} A(\vec{r}) \partial_{x} K_{1}(\vec{r})}{A(\vec{r})}+ \\
& +\left(\frac{\partial_{x} C(\vec{r})}{C(\vec{r})}+\frac{\partial_{x} A(\vec{r})}{A(\vec{r})}-\frac{\partial_{x z}^{2} A(\vec{r})}{\partial_{z} A(\vec{r})}\right) \partial_{z} K_{1}(\vec{r})=0 \\
\partial_{y z}^{2} K_{1}(\vec{r}) & +\left(\frac{\partial_{z} B(\vec{r})}{B(\vec{r})}+\frac{\partial_{z} A(\vec{r})}{A(\vec{r})}-\frac{\partial_{y z}^{2} A(\vec{r})}{\partial_{y} A(\vec{r})}\right) \partial_{y} K_{1}(\vec{r})+ \\
& +\left(-\frac{\partial_{y} A(\vec{r}) \partial_{z} B(\vec{r})}{\partial_{z} A(\vec{r}) B(\vec{r})}+\frac{\partial_{y} A(\vec{r})}{A(\vec{r})}\right) \partial_{z} K_{1}(\vec{r})=0
\end{aligned}
$$

если выполнены следующие соотношения:

$$
\begin{gathered}
\partial_{y} K_{1}(\vec{r})-\frac{\partial_{y} A(\vec{r})\left[K_{2}(\vec{r})-K_{1}(\vec{r})\right]}{A(\vec{r})}=0, \\
\partial_{z} K_{1}(\vec{r})+\frac{\partial_{z} A(\vec{r})\left[-K_{3}(\vec{r})+K_{1}(\vec{r})\right]}{A(\vec{r})}=0, \\
\partial_{x} K_{2}(\vec{r})+\frac{\partial_{x} B(\vec{r})\left[K_{2}(\vec{r})-K_{1}(\vec{r})\right]}{B(\vec{r})}=0, \\
\partial_{z} K_{2}(\vec{r})+\frac{\partial_{z} B(\vec{r})\left[K_{2}(\vec{r})-K_{3}(\vec{r})\right]}{B(\vec{r})}=0
\end{gathered}
$$




$$
\begin{gathered}
\partial_{x} K_{3}(\vec{r})-\frac{\partial_{x} C(\vec{r})\left[-K_{3}(\vec{r})+K_{1}(\vec{r})\right]}{C(\vec{r})}=0, \\
\partial_{y} K_{3}(\vec{r})-\frac{\partial_{y} C(\vec{r})\left[K_{2}(\vec{r})-K_{3}(\vec{r})\right]}{C(\vec{r})}=0 .
\end{gathered}
$$

ЗАмЕчаниЕ 6. Аналогичные уравнения могут быть выписаны и для компонент $K_{2}$ и $K_{3}$. Для величины $K_{2}(\vec{r})$, например, получаем

$$
\begin{aligned}
\partial_{x y}^{2} K_{2}(\vec{r}) & +\frac{\partial_{x} B(\vec{r}) \partial_{y} K_{2}(\vec{r})}{B(\vec{r})}+ \\
+ & \left(\frac{\partial_{y} B(\vec{r})}{B(\vec{r})}+\frac{\partial_{y} A(\vec{r})}{A(\vec{r})}-\frac{\partial_{x y}^{2} B(\vec{r})}{\partial_{y} B(\vec{r})}\right) \partial_{x} K_{2}(\vec{r})=0, \\
\partial_{x z}^{2} K_{2}(\vec{r}) & +\left(\frac{\partial_{z} B(\vec{r})}{B(\vec{r})}-\frac{\partial_{x} C(\vec{r}) \partial_{z} B(\vec{r})}{C(\vec{r}) \partial_{x} B(\vec{r})}\right) \partial_{x} K_{2}(\vec{r})+ \\
+ & \left(\frac{\partial_{x} B(\vec{r})}{B(\vec{r})}-\frac{\partial_{x z}^{2} B(\vec{r})}{\partial_{z} B(\vec{r})}+\frac{\partial_{x} C(\vec{r})}{C(\vec{r})}\right) \partial_{z} K_{2}(\vec{r})=0, \\
\partial_{y z}^{2} K_{1}(\vec{r}) & +\frac{\partial_{z} B(\vec{r}) \partial_{z} K_{2}(\vec{r})}{B(\vec{r})}+ \\
+ & \left(\frac{\partial_{y} C(\vec{r})}{C(\vec{r})}+\frac{\partial_{y} B(\vec{r})}{B(\vec{r})}-\frac{\partial_{y z}^{2} B(\vec{r})}{\partial_{z} B(\vec{r})}\right) \partial_{z} K_{2}(\vec{r})=0 .
\end{aligned}
$$

Важно отметить, что уравнения (22) и (23) представляют собой линейные системы уравнений с условиями совместности в виде системы Дарбу (2)-(4). Это свойство будет в дальнейшем использовано для изучения решений полной системы уравнений Ламе (2)-(7).

Представим теперь некоторые решения системы (21).

Заметим, что из системы (21) вытекают соотношения

$$
K_{i}(\vec{r})\left(\phi_{j}-\phi_{l}\right)+K_{j}(\vec{r})\left(\phi_{l}-\phi_{i}\right)+K_{l}(\vec{r})\left(\phi_{i}-\phi_{j}\right)=0, \quad i \neq j \neq l,
$$

где $\phi_{i}=\phi_{i}\left(x^{i}\right)$ - произвольные зависящие от переменной $x^{i}$ функции. Это ограничительное условие вместе с уравнениями (21) приводит к системе уравнений на функции $K_{i}(\vec{r})$.

Для величины $K_{1}(\vec{r})$, например, получаем

$$
\begin{aligned}
\partial_{x y}^{2} K_{1}(\vec{r})-\frac{1}{2} \frac{-\partial_{x} K_{1}(\vec{r}) b^{\prime}(y)+3 a^{\prime}(x) \partial_{y} K_{1}(\vec{r})}{a(x)-b(y)} & =0 \\
\partial_{x z}^{2} K_{1}(\vec{r})-\frac{1}{2} \frac{3 a^{\prime}(x) \partial_{z} K_{1}(\vec{r})-c^{\prime}(z) \partial_{x} K_{1}(\vec{r})}{a(x)-c(z)} & =0 \\
\partial_{y z}^{2} K_{1}(\vec{r})-\frac{1}{2} \frac{-\partial_{y} K_{1}(\vec{r}) c^{\prime}(z)+\partial_{z} K_{1}(\vec{r}) b^{\prime}(y)}{b(y)-c(z)} & =0
\end{aligned}
$$


где $a(x), b(y), c(z)$ - произвольные функции.

Используя уравнения (22), можно показать, что компоненты метрики пространства Дарбу при данных условиях на главные кривизны суть

$$
\begin{aligned}
A(\vec{r}) & =\frac{U(x)}{\sqrt{a(x)-b(y)} \sqrt{a(x)-c(z)}}, \\
B(\vec{r}) & =\frac{V(y)}{(a(x)-b(y))^{5 / 2} \sqrt{b(y)-c(z)}}, \\
C(\vec{r}) & =\frac{W(z)}{(a(x)-c(z))^{5 / 2} \sqrt{b(y)-c(z)}} .
\end{aligned}
$$

\section{Список литературы}

[1] В. С. Дрюма. Проективные свойства "слабо коммутирующих" семейств операторов. В сб.: IX Всесоюзная геометрическая конференция (Кишинев, 20-22 сентября 1988 г.). Тезисы сообщений. Ред. А. М. Заморзаев. Кишинев: Штиинца, 1988. С. 104-105.

[2] В. С. Дрюма. Математические исследования. 1992. Т. 124. С. 54-68; ТМФ. 1993. T. 99. № 2. C. 241-249.

[3] V. Zakharov. Duke Math. J. 1998. V. 94. № 1. P. 103-139.

[4] T. Wolf. J. Math. Phys. 1986. V. 27. № 9. P. 2354-2359.

[5] R. Jackiw. A pure cotton kink in a funny place. math-ph $/ 0403044$.

[6] E. M. Paterson, A. G. Walker. Quart. J. Math. (Oxford Ser.). 1952. V. 3. P. 19-28; V. Dryuma. Mat. Fiz. Anal. Geom. 2003. V. 10. № 3. P. 1-19; The Riemann and Einstein-Weyl geometries in the theory of ODE's, their applications and all that. In: New Trends in Integrability and Partial Solvability. Proc. of the NATO Advanced Research Workshop (Cadiz, Spain, June 12-16, 2002). NATO Sci. Ser. II: Math., Phys., Chem. V. 132. Eds. A. B. Shabat et al. Dordrecht: Kluwer, 2004. P. 115-156; В. Дрюма. ТМФ. 2001. Т. 128. № 1. С. 15-26; nlin: SI/0303023.

[7] В. Собчук. Тр. семин. вектор. тензор. анал. 1968. Т. 15. С. 65-76.

[8] V. Dryuma. Bul. Acad. Stiinte Repub. Mold. Mat. 1998. V. 1. № 26. P. 55-68. 